\title{
Targeted Therapy in Metastatic Bladder Cancer: Present Status and Future Directions
}

\author{
Mathijs Scholtes ${ }^{1}$, Maryam Akbarzadeh ${ }^{2,3}$, Ellen Zwarthoff ${ }^{4}$, Joost Boormans ${ }^{1}{ }^{1}$, \\ Tokameh Mahmoudi ${ }^{2}$ and Tahlita Zuiverloon ${ }^{1, *}$ \\ 1 Department of Urology, Erasmus MC Cancer Institute, University Medical Center Rotterdam, \\ 3015 GD Rotterdam, The Netherlands; m.scholtes@erasmusmc.nl (M.S.); j.boormans@erasmusmc.nl (J.B.) \\ 2 Department of Biochemistry, Erasmus MC Cancer Institute, University Medical Center Rotterdam, \\ 3015 GD Rotterdam, The Netherlands; m.akbarzadeh@erasmusmc.nl (M.A.); \\ t.mahmoudi@erasmusmc.nl (T.M.) \\ 3 Stem Cell and Regenerative Medicine Center of Excellence, Tehran University of Medical Sciences, \\ 14155-6559 Tehran, Iran \\ 4 Department of Pathology, University Medical Center Rotterdam, 3015 GD Rotterdam, The Netherlands; \\ e.zwarthoff@erasmusmc.nl \\ * Correspondence: t.zuiverloon@erasmusmc.nl
}

Received: 4 September 2020; Accepted: 28 September 2020; Published: 13 October 2020

\begin{abstract}
The recommended treatment for metastatic urothelial carcinoma (mUC) patients is platinum-based chemotherapy. Although initial response rates are moderate, the vast majority of patients experience a relapse due to chemoresistance and eventually succumb to their disease. Furthermore, platinum-based chemotherapy is toxic and approximately $30 \%$ of mUC patients are unfit for chemotherapy. Thus, there is a clear unmet need for novel, more efficacious treatment options in mUC with a safer toxicity profile. To propel the advancement of novel treatment options, we present a summary of key signaling pathways and molecular mechanisms that are known to be involved in bladder cancer tumorigenesis with a focus on promising candidate druggable molecular targets and innovative targeted therapies currently under clinical investigation. Targetable alterations were mainly described in fibroblast growth factor receptor (FGFR) and epidermal growth factor receptor (ErbB) tyrosine kinase receptor (RTK) families, downstream pathways, and chromatin remodelers, which are major bladder cancer driver genes. Drugs targeting the FGFR family members are emerging as personalized treatment options for selected mUC patients with tumor-specific FGFR alterations. The pan-FGFR inhibitor, erdafitinib, was first-in-class to receive U.S. Food and Drug Administration (FDA) approval in 2019, while inhibitors of ErbB family members have shown less potential. Antibody-drug conjugates (ADCs) are a class of targeted therapeutics that deliver cytotoxic drugs in close proximity to cancer cells by targeting RTKs or other transmembrane proteins. Enfortumab vedotin is the first-in-class ADC that was FDA approved for the treatment of locally advanced or mUC in 2019.
\end{abstract}

Keywords: muscle-invasive bladder cancer; tyrosine kinase receptor; RTK; targeted therapy; personalized medicine

\section{Introduction}

Bladder cancer (BC) is a major source of morbidity and mortality, with an estimated 430,000 new cases and 165,000 deaths in 2012 [1]. Tobacco smoke is the single most important risk factor associated with BC [2]. Bladder cancer is almost always of urothelial origin, referred to as urothelial carcinoma of the bladder. Three-quarters of BC patients have non-muscle invasive disease, which is treated by transurethral tumor resection (TUR) followed by adjuvant intravesical instillations. Muscle-invasive 
bladder cancer (MIBC) is a life-threatening disease requiring aggressive treatment due to occult micrometastasis. The recommended treatment option for non-metastatic MIBC is cisplatin-based neoadjuvant chemotherapy (NAC) followed by surgical removal of the bladder (radical cystectomy). In patients who want to keep their bladder, external beam radiotherapy together with concurrent chemotherapy is a feasible alternative. Despite this extensive treatment, approximately half of the MIBC patients who are treated with curative intent will progress to metastatic urothelial carcinoma (mUC). The first-line treatment option in mUC is cisplatin-based chemotherapy, which includes combinations of gemcitabine + cisplatin (gem/cis) or (dose-dense) methotrexate + vinblastine + doxorubicin + cisplatin (ddMVAC), with associated objective response rates (ORRs) of $46 \%$ and $49 \%$, respectively [3]. The long-term survival benefits of gem/cis and ddMVAC regimens are equal, with a median survival of 14 months [3]. Nonetheless, $30 \%$ of mUC patients are cisplatin-ineligible due to having a poor performance status, renal dysfunction, or other comorbidities. Cisplatin-ineligible patients are treated with carboplatin-containing chemotherapy, which has a lower anti-cancer effect but a more favorable toxicity profile than cisplatin [4]. In recent years, immune checkpoint inhibition (ICI) has shown its superior efficacy in mUC patients that relapsed after platinum-based therapy (platinum-relapsed) compared to second-line chemotherapy [5]. The U.S. Food and Drug Administration (FDA)-approved second-line treatment options pembrolizumab or atezolizumab, for treatment of $\mathrm{mUC}$ patients, which were associated with ORRs of $\sim 20 \%[5,6]$. Although the response rates to ICI are low and the median overall survival benefit for all-comers is limited, it is important to emphasize that the majority of responses to ICI are durable, with a median duration of response to atezolizumab of 16 months, while $68 \%$ of responses to pembrolizumab lasted over 12 months [5,6]. For mUC patients that experience a relapse after ICI, no standard treatment options are available. Recent gene expression studies of MIBC identified six molecular subtypes but novel candidates for targeted therapies were not found. These MIBC molecular subtypes are luminal papillary $(24 \%)$, luminal nonspecified $(8 \%)$, luminal unstable $(15 \%)$, stroma-rich $(15 \%)$, basal/squamous $(35 \%)$, and neuro-endocrine-like (3\%) [7]. The Cancer Genome Atlas (TCGA) project showed that approximately $69 \%$ of MIBC patients harbored molecular alterations that could be targeted for therapy [8-10]. The multiomics approach of TCGA combined gene-expression profiling with whole-exome sequencing, RPPA protein expression, and DNA methylation that culminated into a snapshot of the molecular landscape of MIBC [8-10]. Frequently occurring alterations were identified in the receptor tyrosine kinase (RTK)/MAPK pathway, (RTK)/PI3K/AKT/mTOR pathway, and chromatin-modifying genes. Identification of BC driver genes provides an opportunity to develop and investigate personalized treatment options for UC, and as a result, the study of mUC is rapidly evolving. Recently, the FDA has granted accelerated approval for erdafitinib, a first-in-class pan-FGFR (fibroblast growth factor receptor inhibitor, and enfortumab vedotin, a first-in-class antibody-drug conjugate (ADC). The aim of this review was to provide a contemporary overview of targeted therapy in mUC. We address clinical trials that are currently being conducted in $\mathrm{mUC}$ patients and highlight future promising candidates for novel treatment options.

\section{Methods}

A literature search was conducted using the Embase, Medline, Web of Science Core Collection, Cochrane Central Register of Controlled Trials, and Google Scholar databases for results on urothelial cancer and tyrosine kinase inhibitors published from 1988 until March 2020. The Embase search was as follows: "('bladder cancer'/exp OR 'transitional cell carcinoma'/de OR 'ureter carcinoma'/de OR (((bladder* OR transition*-cell* OR urothelial OR ureter* OR uretal) NEAR/3 (cancer* OR neoplas* OR malign* OR carcinom* OR metast* OR tumor* OR tumour*))):ab,ti,kw) AND ('protein tyrosine kinase inhibitor'/exp OR 'epidermal growth factor receptor kinase inhibitor'/exp OR (((tyrosin* OR epidermal-growth* OR EGF) NEAR/3 (kinas*) NEAR/3 (inhibitor*))". The search resulted in 4888 full-text articles and/or congress papers published in the English language, including 1293 duplicates. After the removal of duplicates, 3595 results were screened by title and abstract. Eventually, 591 studies were grouped according to pre-determined subjects: (1) FGFR family, (2) epidermal growth factor 
receptor (ErbB) family, (3) MAPK pathway, (4) PI3K pathway, and (5) unspecified. However, 512 of the selected publications proved irrelevant and were excluded upon assessment of the full-text articles because of low-level evidence (case series, expert opinions, comments on other articles), or because congress papers and interim analysis became outdated when studies were published as primary research articles. Ultimately, 79 articles were included in this review.

\section{Pharmacological Inhibition of Fibroblast Growth Factor Receptor Signaling}

RTKs and their downstream signaling pathway members are frequently mutated in treatment-naive MIBC [8-10]. Whole-exome sequencing of 131 MIBC patients identified molecular alterations in the RTK/MAPK pathway in $45 \%$ of cases and the PI3K/AKT/mTOR pathway in $42 \%$ of cases [8-10]. Both MAPK and PI3K/AKT/mTOR pathways can be activated by the same RTKs, as illustrated in Figure 1 [11]. RTKs contain an extracellular ligand-binding domain, a transmembrane domain, and an intracellular tyrosine-kinase domain. Ligand binding causes RTKs to dimerize and become activated. Constitutively activated RTK signaling can occur in the presence of missense mutations, gene fusions, and increased RTK protein expression through altered gene expression or gene amplifications. RTKs involved in UC are primarily members of the FGFR family (FGFR1, FGFR2, FGFR3, and FGFR4) and members of the ErbB receptor family [8-10].

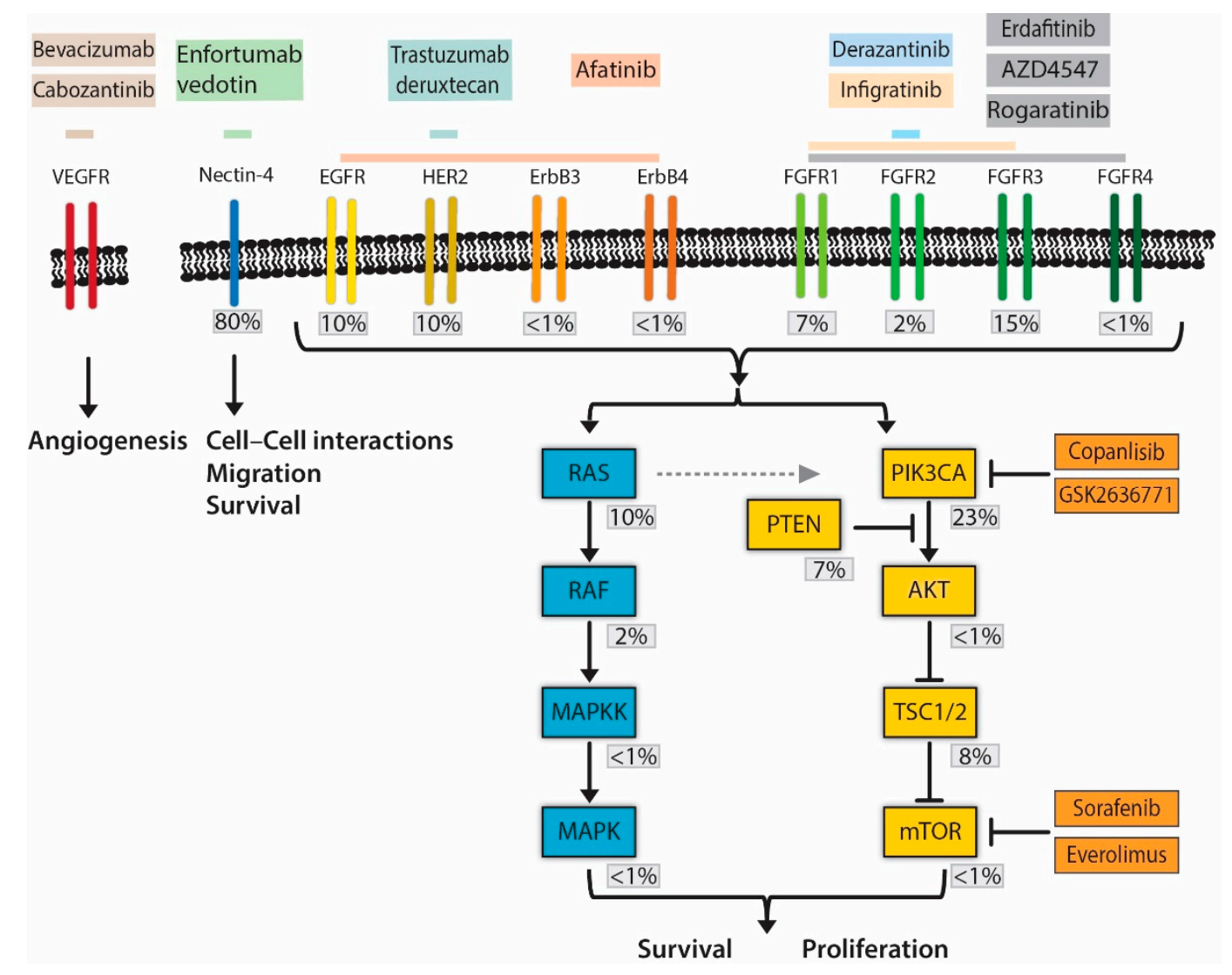

Figure 1. Therapeutic targets in muscle-invasive bladder cancer (MIBC). Schematic overview of (RTK)/MAPK (blue) and (RTK)/PI3K/AKT/mTOR (yellow) pathway alterations that are frequently detected in MIBC patients. Gene alteration frequencies are indicated as percentages under the corresponding proteins. Therapeutic agents that target particular or multiple RTKs are shown above their corresponding targets. Therapeutic agents targeting PIK3CA or mTOR (orange) are depicted next to their targets.

Approximately $11-12 \%$ of MIBC patients undergoing a cystectomy have activated FGFR3 mutations $[8,12]$. Of these mutations, $95 \%$ are in the extracellular or transmembrane domain and result 
in an amino acid changing into a cysteine. This causes the formation of disulfide bridges between two receptor molecules and the constitutive activation of the receptors [13]. FGFR3-activating mutations were detected in $8 / 27$ superficial tumor areas from patients with MIBC, yet only 4 of these 8 mutations were also present in the muscle-invasive front [12]. In addition, $5 \%$ of the lymph node metastases contained FGFR3 mutations matching those detected in the primary tumor [12]. Future studies should include multiple samples from papillary (superficial) and invasive tumor areas to confirm initial findings that imply a decline in FGFR3 mutation frequencies as tumors progress. Nonetheless, the corresponding FGFR3 mutations between primary MIBC tumors and lymph node metastases provide a rationale for systemic FGFR3-directed therapy.

In addition to FGFR3 mutations, $30-40 \%$ of patients with MIBC have FGFR3 overexpression $[14,15]$. However, FGFR3 overexpression has no effect on clinical outcome in MIBC patients treated with radical cystectomy [16]. FGFR3 gene amplifications (3\%) and FGFR3 gene fusions $(2 \%)$ are rare events in MIBC [8-10].

FGFR1 amplifications are the most frequently detected FGFR1 alterations and are reported in $7 \%$ of UC patients [17]. In addition to gene amplifications, alternative splicing of FGFR1 is associated with increased tumor stage and grade [18]. Interestingly, FGFR1 overexpression induced epithelial-to-mesenchymal transition (EMT) in UC cell lines $[19,20]$. FGFR2 gene fusions have been described in 2-3\% of BC patients [17]. Thus, molecular alterations in FGFR1, FGFR2, and FGFR3 have been described as cancer driver genes in mUC. Patient tumors can simultaneously harbor multiple FGFR alterations [8-10]. In addition, tumor cells can upregulate one FGFR in response to inhibition of another. The presence of multiple synchronous FGFR-alterations and resistance mechanisms provide a rationale for combined inhibition of FGFR1, FGFR2, and FGFR3. Recently, several therapeutic agents targeting multiple FGFRs have been investigated (Table 1). Infigratinib (BGJ-398) is an FGFR1-3 inhibitor with response rates of $40-50 \%$ in metastatic upper tract urothelial carcinoma (UTUC) patients with FGFR3 mutations or fusions [21,22]. Adjuvant treatment of MIBC/UTUC with infigratinib is currently under investigation in the phase III PROOF 302 trial (NCT04197986). AZD4547 is an inhibitor of FGFR1/2/3, tyrosine-protein kinase KIT (c-KIT), and colony-stimulating factor 1 receptor (CSF1R) with weaker activity against FGFR4 [23]. AZD4547 is currently being investigated in the BISCAY trial (NCT02546661) as part of a biomarker-directed, multidrug phase Ib open-label trial for treatment-relapsed mUC patients. AZD4547 treatment is also examined in the biomarker-directed MATCH Screening Trial (NCT02465060), which is a basket trial including mUC patients. Patients harboring somatic FGFR1-3 mutations or translocations, determined by DNA sequencing of tumor biopsies, will receive AZD4547 treatment until disease progression or unacceptable toxicity occurs. Rogaratinib (BAY1163877) is an FGFR1-4 inhibitor that was found to be well tolerated in a phase I/II clinical trial [24]. Rogaratinib is currently under investigation as a second-line treatment in patients with locally advanced or mUC expressing FGFR1/3 mRNA, identified by in situ hybridization of archival tissue, in the FORT-1 trial (NCT03410693). Derazantinib is a selective FGFR2 inhibitor that is being investigated as a single treatment or in combination with atezolizumab as a first- or second-line treatment in the FIDES-02 trial, which enrolled an estimated 303 patients with mUC harboring genetic FGFR aberrations (NCT04045613). Erdafitinib, a pan-FGFR inhibitor, showed promising results due to its limited toxicity, an overall response rate (ORR) of $40 \%$, and a complete response rate (CR) of $3 \%$ in mUC patients with FGFR3 alterations [25]. Based on these results, erdafitinib was granted accelerated FDA approval as a second-line treatment for mUC patients with FGFR alterations. Nonetheless, it remains to be determined whether mUC patients with FGFR1 or FGFR3 overexpression or alternative splicing of FGFR1 benefit from FGFR inhibitors. 
Table 1. Targeted therapies that are currently being investigated in MIBC.

\begin{tabular}{ccc}
\hline Drug & Target & Clinical Trials \\
\hline erdafitinib & FGFR1-4 & NCT03473743, NCT02465060 (MATCH) \\
infigratinib (BGJ-398) & FGFR1-3 & NCT04197986 (PROOF 302), \\
AZD4547 & FGFR1-4, Kit, CSF1R & NCT02546661 (BISCAY), NCT02465060 \\
(MATCH) \\
rogaratinib (BAY 1163877) & FGFR1-4 & NCT03410693 (FORT-1) \\
derazantinib (ARQ 087) & FGFR2 & NCT04045613 (FIDES-02) \\
afatinib (BIBW2992) & ErbB1-4 & NCT02780687 (LUX Bladder-1) \\
trastuzumab deruxtecan (DS-8201a) & Erbb2 & NCT03523572 \\
cabozantinib (XL184) & VEGFR, c-MET, AXL, RET & NCT01688999, NCT03866382, NCT03824691 \\
(ARCADIA), NCT03170960 (COSMIC-021) \\
copanlisib & mTOR & NCT02465060 (MATCH) \\
GSK2636771 & mTOR & NCT02465060 (MATCH) \\
valemetostat (DS3201) & EZH2 & NCT04388852 \\
rucaparib & EZH2 & NCT03854474 \\
olaparib & PARP1 & NCT03397394 (ATLAS) \\
entinostat & PARP1 & NCT03375307, NCT02546661 (BISCAY) \\
\hline
\end{tabular}

ARCADIA: (hARnessing CAbozantinib and Durvalumab Immuno-oncology Association, c-MET: MET proto-oncogene, CSF1R: Colony stimulating factor 1 receptor, ErbB: epidermal growth factor receptor, EZH2: Enhancer of zeste homolog 2, FGFR: fibroblast growth factor receptor, HDAC1: Histone deacetylase 1, MATCH: Molecular Analysis for Therapy Choice, mTOR: mammalian target of rapamycin, PARP: Poly(ADP-ribose) polymerase 1 RET: Ret proto-oncogene, VEGFR: vascular endothelial growth factor receptor.

\section{Targetting ErbB Receptor Family Signaling}

The ErbB family consists of four RTKs: epidermal growth factor receptor (EGFR, also called HER-1 or ErbB-1), HER2 (also called ErbB-2 or HER-2/Neu), HER3 (ErbB3), and HER4 (ErbB4) [26]. Of these, EGFR and HER-2 have been implicated in BC.

EGFR gene amplifications have been described in $9-14 \%$ of MIBC cases $[9,27]$. EGFR expression is enriched in the basal/squamous subtype $[7,27]$. Likewise, the activity of the EGFR regulon is also increased in basal/squamous tumors $[7,28]$. The basal/squamous expression subtype is a major MIBC subtype, found in approximately $35 \%$ of MIBC patients and is associated with poor outcome after radical cystectomy [7]. Despite upregulated EGFR mRNA expression and increased activity of the EGFR regulon in basal/squamous MIBC, the EGFR targeting antibody cetuximab did not show any additional benefit over gem/cis in patients with $\mathrm{mUC}$, irrespective of the EGFR status [29].

HER2 is amplified or mutated in $9 \%$ of MIBC cases [8-10,30]. A study of 90 MIBC patients treated with cystectomy showed that HER2 overexpression detected using immunohistochemistry (IHC) was associated with a lower recurrence-free and cancer-specific survival [31]. In contrast, a larger study that compared two primary tumor samples with two matched lymph node metastasis from 137 patients using fluorescence in situ hybridization (FISH) and IHC could not confirm these findings; while HER2 amplifications in primary tumors were associated with lower overall survival, there was poor concordance between the HER2 gene amplifications (using FISH) and protein expression (using IHC) [30]. Interestingly, a higher frequency of HER2 amplifications was described in lymph node metastases compared to primary MIBC tumor samples (15.3\% vs. 8.7\%) [30]. Thus, while HER2 amplifications seem to be associated with a poor prognosis and more frequent tumor progression, the prognostic implications of HER2 overexpression are currently unclear. Both HER2 amplification and overexpression are well-validated biomarkers for anti-HER2 targeted therapy in breast and gastric cancers but clinical trials investigating HER2 inhibition in MIBC patients with HER2 or EGFR alterations have not been promising. Treatment with lapatinib, an EGFR/HER2 specific small-molecule inhibitor, showed no efficacy over that of a placebo in a phase III trial in mUC patients with positive EGFR and HER2 expression [32]. Furthermore, HER2 inhibition with the monoclonal antibody trastuzumab in conjunction with gem/cis or gemcitabine and carboplatin (gem/carbo) did not yield additional clinical benefit in mUC patients with HER2 overexpression, compared to chemotherapy alone [33]. 
Biological differences between MIBC and breast cancer might provide an explanation why HER2-targeted therapy has not been as successful in MIBC as in breast cancer. Differences in response to HER2-targeted therapy observed between breast cancer and $\mathrm{mUC}$ patients might be explained by dissimilar HER-2 mutations driving tumor growth. HER2 alterations are equally found in TCGA MIBC and breast cancer datasets, although MIBC is characterized by more point mutations and fewer gene amplifications [9]. Putative activating mutations in the extracellular domain of HER2 are described as being present in $40 \%$ of the histological variant micropapillary UC compared to 5-9\% of non-micropapillary UC [34,35]. Micropapillary disease is a rare histological subtype present in around $2 \%$ of MIBC patients and is associated with a poor prognosis [36]. The association between putative HER2 activating mutations, worse prognosis and a particular histological variant suggests driver effects of putative HER2 activating mutations. On the other hand, HER2 amplifications are most frequently detected in the MIBC subtype identified as "genomically unstable" [27]. This subtype is associated with worse prognoses and is characterized by frequent chromosomal aberrations that can lead to copy-number alterations (CNAs). Due to frequent CNAs detected in this subtype, it has been hypothesized that HER2 amplifications, in the presence of many other large chromosomal aberrations, are possibly passenger effects in MIBC [37]. Thus, patient selection based on HER2 amplifications or IHC alone might not be the best method for selecting MIBC patients for HER2-targeted therapy [27,37]. Currently, the LUX Bladder-1 trial is investigating monotherapy with the ERB1-4 inhibitor afatinib in platinum-relapsed mUC patients with either HER2 or HER3 mutations, HER2 amplifications, or EGFR amplifications (NCT02780687).

In summary, HER2 and EGFR inhibitory therapy have been evaluated in MIBC with initially disappointing results, which was potentially due to an inadequate patient selection process that focused on HER2 amplifications and/or overexpression instead of activating mutations. Future studies on the targetability of HER2 and EGFR should not neglect the molecular landscape of MIBC, which suggests that patient selection should be based on activated HER2 mutations (micropapillary variant histology) and HER2 or EGFR regulon activity as interesting candidates for ErbB inhibitory therapy.

\section{MAPK and Pi3K/AKT/mTOR Pathway Alterations}

The MAPK and Pi3K/AKT/mTOR signaling cascades are activated by RTKs. Activation of MAPK and PI3K signaling cascades is a common finding in BC and promotes cell growth and survival [38]. These pathways show frequent crosstalk by sharing subunits and negative/positive feedback loops [38]. Activating mutations in RAS genes-HRAS (5\%), KRAS (5\%), or NRAS (2\%)-are present in a minority of MIBC patients [9]. The Pi3K/Akt/mTOR pathway can also be directly activated by activating mutations in the phosphoinositide 3-kinase catalytic subunit alpha (PIK3CA). Constitutively active PIK3CA produces phosphatidylinositol-3-phosphate (PIP3), which activates downstream actors of the Pi3K/Akt/mTOR pathway. Approximately 22\% of MIBC patients have such activating PIK3CA mutations [8]. Other genes commonly mutated in the Pi3K/AKT/mTOR pathway are the tumor suppressor genes PTEN and TSC1. PTEN is a phosphatase that antagonizes Pi3K by breaking down PIP3 and is mutated in 3\% of MIBC patients, while TSC1 inhibits the pathway further downstream and is mutated in $8 \%$ of MIBC patients [8]. The MAPK and Pi3K/AKT/mTOR pathways are interesting candidates for targeted therapy, as more than $25 \%$ of patients have alterations in one of these pathways. However, crosstalk between the MAPK and Pi3K/AKT/mTOR pathways and upstream RTKs provide cancer cells with effective mechanisms to escape treatment directed against one pathway via activation of the other [38-43]. For example, cell lines upregulate EGFR expression to overcome a temporary decrease in MAPK signaling induced by FGFR inhibition [41]. In addition, downstream activation of the $\mathrm{Pi} 3 K / A K T / m T O R$ pathway has been described as a resistance mechanism to FGFR inhibition $[40,42,43]$. Thus, these findings prompt development of a method that produces a combined inhibition of the RTK-MAPK and RTK-Pi3K/AKT/mTOR pathways.

A phase I study investigating the mTOR inhibitor everolimus combined with the vascular endothelial growth factor RTK (VEGFR) inhibitor pazopanib showed a favorable toxicity profile and 
clinical benefit was observed in 4 of 19 included patients with mUC harboring TSC1/TSC2 mutations [44]. Two PIK3CA inhibitors are also included in the MATCH basket trial: copanlisib (BAY 80-6946) for patients with Pi3KCA-activating mutations and or PTEN-inactivating mutations, and GSK2636771 for patients with PTEN-inactivating mutations (NCT02465060). The mTOR inhibitor sorafenib was investigated in the neoadjuvant setting in combination with gem/cis and showed acceptable toxicity; furthermore, the ErbB family or MAPK pathway alterations were predictive of therapy response [45]. Sorafenib combined with vinflunine in platinum-relapsed mUC patients showed an impressive ORR of $41 \%$, supporting further trials [46]. In summary, RTKs converge on the MAPK and Pi3K/AKT/mTOR pathways to drive MIBC. Crosstalk between these pathways provides UC cells with mechanisms of resistance to RTK inhibition. Treatment resistance could be overcome by either the combined inhibition of multiple RTKs at the same time or interference with the crosstalk between the two pathways in pre-clinical models. We are looking forward to subsequent clinical trials that evaluate the effect of FGFR and ErbB family inhibition by, for example, afatinib + erdafitinib administered synchronously or in short intervals. Likewise, future clinical trials should consider FGFR or ErbB inhibition combined with Pi3K or mTOR inhibitors.

\section{Targeting UC Vascularization with VEGF Inhibitors}

Solid tumors depend on adequate blood supply in order to grow and metastasize. In the case of hypoxia, cells release vascular endothelial growth factor (VEGF), which activates the VEGFR expressed on vascular endothelial cells, thereby stimulating neovascularization [47]. VEGF or VEGFR have been targeted in several tumor types to inhibit the formation of new blood vessels, thus impairing tumor growth [48]. VEGF/VEGFR inhibition is not tumor-specific and there are currently no biomarkers available to select patients with an increased probability of response to therapy. Thus, VEGF/VEGFR inhibition can currently be classified as a form of non-personalized, targeted therapy. Several VEGF/VEGFR-directed therapies have been evaluated in MIBC. Bevacizumab is an antibody that binds VEGF, preventing the activation of VEGFR. Bevacizumab has been investigated in combination with gem/cis as a first-line treatment option in mUC and showed an ORR of $72 \%$ in a phase II trial [49]. Despite these promising initial results, gem/cis + bevacizumab did not increase the overall survival in a follow-up phase III trial [50], demonstrating the importance of confirming phase II ORR results in phase III randomized trials that evaluate overall survival. Ongoing biomarker studies could identify subsets of patients who benefit from anti-angiogenic therapy. Cabozantinib is a small molecule inhibitor of VEGFR2 that also inhibits other RTKs (c-MET, AXL, and RET) [51]. Cabozantinib treatment for mUC is currently under investigation as monotherapy in a third-line setting (NCT01688999). Another study investigated cabozantinib + nivolumab + ipilimumab and demonstrated a favorable toxicity profile [52,53]. A phase II trial is currently being performed to evaluate its efficacy (NCT03866382). The ARCADIA (hARnessing CAbozantinib and Durvalumab Immuno-oncology Association) phase II trial investigates the efficacy of a cabozantinib + durvalumab combination therapy in patients with platinum-relapsed mUC (NCT03824691) $[54,55]$. The safety and tolerability of cabozantinib + atezolizumab are being investigated in the COSMIC-021 phase Ib trial (NCT03170960) that includes patients with locally advanced or metastatic UC, among other tumor types [56-58]. Preliminary results based on the castration-resistant prostate cancer cohort point to a tolerable safety profile for the treatment combination [59].

The inhibition of neovascularization by blocking VEGF or VEGFR has shown favorable toxicity profiles that allow for combination therapy with gem/cis or ICI. Anti-VEGF therapy + gem/cis has not shown a survival benefit despite an impressive ORR of $72 \%$ in a phase II trial. Results of clinical trials that combine VEGFR-inhibition with ICI are currently pending and the value of an anti-VEGF/VEGFR treatment for $\mathrm{MUC}$ is yet unclear. Nonetheless, favorable toxicity profiles support the future evaluation of anti-VEGF/VEGFR therapy combined with drugs other than gem/cis or ICI. 


\section{Promising Targeted Treatment Approach: Antibody-Drug Conjugates}

Antibody-drug conjugates (ADCs) are drugs comprised of a monoclonal antibody tethered to a cytotoxic drug using a linker. ADCs rely on monoclonal antibodies to guide (highly) cytotoxic drugs to the proximity of target cells, whereupon the drug is released. Ideally, the monoclonal antibody component targets antigens that are exclusively and homogeneously expressed on cell membranes of cancer cells [60]. For maximal on-target effects, ADC-targeted antigens are not to be secreted and are ideally internalized upon ADC binding [61,62]. Drugs tethered using non-cleavable linkers are only released following a complete proteasomal breakdown of the antibody component and will remain linked to an amino acid residue derived from the degraded antibody [63]. Cleavable linkers are designed to release drugs in response to particular extracellular and intracellular environments $(\mathrm{pH}$, redox potential) or following enzymatic cleavage [63].

The first ADC that received FDA approval for the treatment of mUC patients was enfortumab vedotin. Enfortumab targets nectin- 4 and is tethered to the cytotoxic agent vedotin using a protease-cleavable linker. FDA approval was based on results of a phase I clinical trial showing a promising ORR of $42 \%$ and a CR of $9 \%$ in mUC [64]. Side-effects most often associated with enfortumab vedotin or other vedotin-based ADCs are fatigue, peripheral neuropathy, rash, and hyperglycemia [65].

Enfortumab vedotin paves the way for other ADCs to be used against MIBC, and ADCs targeting ErbB family members are currently being evaluated. HER2 overexpression in BC has been targeted by ADCs, even though the anticancer effect of HER2 inhibition was found to be minimal. Disitamab vedotin (RC48-ADC) combines the HER2-specific desitamab with vedotin using a protease-cleavable linker and has shown a promising ORR of 60\% in HER2-positive mUC patients that have progressed on prior platinum-based therapy [66,67]. ADCs based on trastuzumab are also being investigated. Trastuzumab deruxtecan (DS-8201a) is trastuzumab tethered to the chemotherapeutic agent deruxtecan via a cleavable peptide linker and is currently being investigated in combination with nivolumab as a second-line therapy in a phase I trial for patients with HER2-positive metastatic breast cancer or mUC (NCT03523572). Common grade 3 adverse events associated with trastuzumab deruxtecan are interstitial lung disease, neutropenia, anemia, or thrombocytopenia. Trastuzumab emtansine (T-DM1) combines trastuzumab with cytotoxic entamsine and has a non-cleavable linker. Common side effects are conjunctivitis, dry eyes, and neutropenia. Trastuzumab entamsine was evaluated in a phase I clinical trial that enrolled patients with multiple tumor types carrying HER2 amplifications but did not yield any response in the three mUC patients available for analysis [68].

In conclusion, HER2-targeted ADCs, like enfortumab vedotin, are a promising new class of drugs that exploit HER2 overexpression, irrespective of the anticancer effect of HER2 inhibition.

Patients with EGFR or HER2 overexpression are an interesting population to further investigate the effect of ADCs. Phase II and phase III clinical trials evaluating EGFR or FGFR-targeted ADCs in mUC are awaited.

\section{Sampling and Trial Setup}

Novel anticancer therapies may target rare molecular alterations. The rare occurrence of these individual driver alterations presents challenges regarding enrolling enough patients with the same driver alterations and tumor type into clinical trials. Basket trials are designed to overcome this challenge by enrolling patients with different tumor types but similar molecular alterations. These trials are based on the assumption that patients with similar driver alterations but different tumor types will have comparable responses to targeted therapy [69]. The histological background of a tumor determines how a patient's cancer cells have evolved and continue to evolve during treatment. For example, patients with FGFR2/3 alterations are more likely to respond to erdafitinib if they have urothelial carcinoma or cholangiocarcinoma instead of other tumor types [70].

A major problem with basket trials is the accrual of enough patients with the same tumor type. This could lead to underpowered evaluations in tumor-type-specific subanalyses. As treatment responses can differ between different tumor types, there is a risk that basket trials could potentially 
lead to early termination of treatment arms and failure to identify (small) patient populations in whom biomarker-guided treatments are effective. Umbrella trials do not have this limitation as only patients with a single tumor type are enrolled. In umbrella trials, patients with the same tumor type are screened for multiple molecular aberrations and subsequently randomized for either the targeted therapy or standard of care. Umbrella trials are flexible, which allows for the closing of ineffective treatment arms and adding treatments without writing a new protocol. The flexibility of umbrella trials could be embraced to efficiently evaluate the safety profiles of novel drugs. Nonetheless, the probability that there is a direct therapeutic benefit to participants is low for both trial designs [69]. Clinical trials that evaluate biomarker-guided targeted therapy can be hampered by several hurdles that need to be overcome. Trials require the accrual of enough patients to allow for subanalyses, acquired samples should be appropriate for biomarker evaluation, and patient samples should be accessible for analyses. Problems with patient accrual can be overcome by intensifying the collaboration in international consortia and designing umbrella trials that include treatment arms for patients without detected biomarkers.

The accurate biomarker prediction of treatment outcomes requires biomarkers to be assessed using a patient's lethal tumor clones. Biomarker accuracy is therefore hampered by tumor heterogeneity. The mutagenic nature of urothelial cancer gives rise to different tumor subclones that continue to evolve during a patient's natural disease course and treatment [71,72]. The mutational variation observed within a primary tumor appears to explain a minor fraction of the total genetic tumor heterogeneity observed between primary and metastatic lesions [73,74]. The vast intratumor heterogeneity is explained by metastatic spread, which is an early event in urothelial cancer evolution and thus few mutations are shared between primary tumor sites and metastatic lesions [72]. Biomarker assessment on primary tumors might therefore not be optimal for predicting a targeted treatment response in patients with mUC. Moreover, the genomic heterogeneity is even greater in patients with multiple primary tumors, further complicating the use of primary tumor samples for targeted treatment allocation [74]. The sampling bias that arises from a primary tumor that does not correspond with metastatic lesions could particularly hamper patient selection for anti-FGFR3 therapy, as FGFR3 mutation frequencies decrease with progression from non-muscle invasive bladder cancer (NMIBC) to MIBC to lymph node metastasis [12]. To conclude, biomarkers that guide targeted therapy are ideally evaluated in patients' most lethal tumor clones. Most mUC patients succumb from metastasis, thus biomarker assessment requires access to metastatic lesions.

Samples from metastatic lesions can be obtained by taking needle biopsies, which is burdensome and puts patients at risk for complications. Liquid biopsies allow for the evaluation of tumor DNA that has shed into the circulation. Tumor DNA is continuously shed into the circulation and can be accessed by drawing blood. The analysis of circulating tumor DNA (ctDNA) using ultra-deep sequencing or digital droplet PCR allows for the early detection of metastatic relapse and monitoring the treatment response in a minimally invasive manner $[75,76]$. Furthermore, actionable molecular alterations, such as FGFR3 hotspot mutations, have been detected in ctDNA $[77,78]$. Nonetheless, it remains to be determined whether a patient's most burdensome tumor clones can be identified as such by evaluating the allele frequencies of ctDNA. Ideally, mutations specific to proliferating or metastasizing tumor clones are indeed represented by greater allele frequencies detected in ctDNA. This would potentially enable umbrella trials that loop back to renewed biomarker assessment and treatment allocation every time that a treatment relapse occurs.

\section{Future Directions}

After years of modest innovation, the clinical practice of treating $\mathrm{mUC}$ is now rapidly changing, driven by the molecular characterization of MIBC. Molecular alterations in RTKs, the MAPK pathway the PI3K/AKT/mTOR pathway, and chromatin remodelers have been identified as potential candidates for targeted therapy. Pan-FGFR inhibitors have proven patient benefits and erdafitinib was the first targeted therapy for mUC that was approved for clinical practice. The clinical efficacy of ErbB family 
inhibition was less promising, but an optimized patient selection and ErbB-directed ADCs are strategies that could improve treatment outcomes. Enfortumab vedotin is the first-in-class ADC to be introduced into clinical practice, although phase III trials are awaited to confirm the patient benefits. Lastly, for future directions, the functions of mutated chromatin remodelers in BC are understudied and their potential for therapeutic targeting in $\mathrm{BC}$ remains to be explored.

Author Contributions: Conceptualization, M.S. and T.Z.; methodology, M.S. and T.Z.; writing—original draft preparation, M.S. and T.Z.; writing-review and editing, M.S., M.A., E.Z., J.B., T.M., and T.Z. All authors have read and agreed to the published version of the manuscript.

Funding: This research received no external funding.

Acknowledgments: The authors wish to thank M.F.M. Engel from the Erasmus MC Medical Library for developing the search strategies.

Conflicts of Interest: The authors declare no conflict of interest.

\section{References}

1. Antoni, S.; Ferlay, J.; Soerjomataram, I.; Znaor, A.; Jemal, A.; Bray, F. Bladder Cancer Incidence and Mortality: A Global Overview and Recent Trends. Eur. Urol. 2017, 71, 96-108. [PubMed]

2. Burger, M.; Catto, J.W.F.; Dalbagni, G.; Grossman, H.B.; Herr, H.; Karakiewicz, P.; Kassouf, W.; Kiemeney, L.A.; La Vecchia, C.; Shariat, S.; et al. Epidemiology and Risk Factors of Urothelial Bladder Cancer. Eur. Urol. 2013, 63, 234-241. [CrossRef] [PubMed]

3. von der Maase, H.; Hansen, S.W.; Roberts, J.T.; Dogliotti, L.; Oliver, T.; Moore, M.J.; Bodrogi, I.; Albers, P.; Knuth, A.; Lippert, C.M.; et al. Gemcitabine and cisplatin versus methotrexate, vinblastine, doxorubicin, and cisplatin in advanced or metastatic bladder cancer: Results of a large, randomized, multinational, multicenter, phase III study. J. Clin. Oncol. 2000, 18, 3068-3077. [CrossRef] [PubMed]

4. Galsky, M.D.; Chen, G.J.; Oh, W.K.; Bellmunt, J.; Roth, B.J.; Petrioli, R.; Dogliotti, L.; Dreicer, R.; Sonpavde, G. Comparative effectiveness of cisplatin-based and carboplatin-based chemotherapy for treatment of advanced urothelial carcinoma. Ann. Oncol. 2012, 23, 406-410. [CrossRef] [PubMed]

5. Bellmunt, J.; de Wit, R.; Vaughn, D.J.; Fradet, Y.; Lee, J.L.; Fong, L.; Vogelzang, N.J.; Climent, M.A.; Petrylak, D.P.; Choueiri, T.K.; et al. Pembrolizumab as Second-Line Therapy for Advanced Urothelial Carcinoma. N. Engl. J. Med. 2017, 376, 1015-1026. [CrossRef]

6. Powles, T.; Duran, I.; van der Heijden, M.S.; Loriot, Y.; Vogelzang, N.J.; De Giorgi, U.; Oudard, S.; Retz, M.M.; Castellano, D.; Bamias, A.; et al. Atezolizumab versus chemotherapy in patients with platinum-treated locally advanced or metastatic urothelial carcinoma (IMvigor211): A multicentre, open-label, phase 3 randomised controlled trial. Lancet 2018, 391, 748-757. [CrossRef]

7. Kamoun, A.; de Reynies, A.; Allory, Y.; Sjodahl, G.; Robertson, A.G.; Seiler, R.; Hoadley, K.A.; Groeneveld, C.S.; Al-Ahmadie, H.; Choi, W.; et al. A Consensus Molecular Classification of Muscle-invasive Bladder Cancer. Eur. Urol. 2020, 77, 420-433. [CrossRef]

8. Robertson, A.G.; Kim, J.; Al-Ahmadie, H.; Bellmunt, J.; Guo, G.; Cherniack, A.D.; Hinoue, T.; Laird, P.W.; Hoadley, K.A.; Akbani, R.; et al. Comprehensive Molecular Characterization of Muscle-Invasive Bladder Cancer. Cell 2017, 171, 540-556.e25. [CrossRef]

9. Weinstein, J.N.; Kim, J.; Creighton, C.J.; Akbani, R.; Hoadley, K.A.; Kim, W.Y.; Morgan, M.B.; Hinoue, T.; Rosenberg, J.E.; Bajorin, D.F.; et al. Comprehensive molecular profiling of urothelial bladder cancer at the DNA, RNA, and protein levels: A TCGA project. J. Clin. Oncol. 2014, 32, 4509. [CrossRef]

10. Cancer Genome Atlas Research Network. Comprehensive molecular characterization of urothelial bladder carcinoma. Nature 2014, 507, 315-322. [CrossRef]

11. Shaw, R.J.; Cantley, L.C. Ras, PI(3)K and mTOR signalling controls tumour cell growth. Nature 2006, 441, 424-430. [CrossRef] [PubMed]

12. Pouessel, D.; Neuzillet, Y.; Mertens, L.S.; van der Heijden, M.S.; de Jong, J.; Sanders, J.; Peters, D.; Leroy, K.; Manceau, A.; Maille, P.; et al. Tumor heterogeneity of fibroblast growth factor receptor 3 (FGFR3) mutations in invasive bladder cancer: Implications for perioperative anti-FGFR3 treatment. Ann. Oncol. 2016, 27, 1311-1316. [CrossRef] [PubMed] 
13. van Rhijn, B.W.; van Tilborg, A.A.; Lurkin, I.; Bonaventure, J.; de Vries, A.; Thiery, J.P.; van der Kwast, T.H.; Zwarthoff, E.C.; Radvanyi, F. Novel fibroblast growth factor receptor 3 (FGFR3) mutations in bladder cancer previously identified in non-lethal skeletal disorders. Eur. J. Hum. Genet. 2002, 10, 819-824. [CrossRef] [PubMed]

14. Tomlinson, D.C.; Baldo, O.; Harnden, P.; Knowles, M.A. FGFR3 protein expression and its relationship to mutation status and prognostic variables in bladder cancer. J. Pathol. 2007, 213, 91-98. [CrossRef]

15. Guancial, E.A.; Werner, L.; Bellmunt, J.; Bamias, A.; Choueiri, T.K.; Ross, R.; Schutz, F.A.; Park, R.S.; O'Brien, R.J.; Hirsch, M.S.; et al. FGFR3 expression in primary and metastatic urothelial carcinoma of the bladder. Cancer Med. 2014, 3, 835-844. [CrossRef]

16. van Rhijn, B.W.G.; Mertens, L.S.; Mayr, R.; Bostrom, P.J.; Real, F.X.; Zwarthoff, E.C.; Boormans, J.L.; Abas, C.; van Leenders, G.J.L.H.; Götz, S.; et al. FGFR3 Mutation Status and FGFR3 Expression in a Large Bladder Cancer Cohort Treated by Radical Cystectomy: Implications for Anti-FGFR3 Treatment? Eur. Urol. 2020. [CrossRef]

17. Helsten, T.; Elkin, S.; Arthur, E.; Tomson, B.N.; Carter, J.; Kurzrock, R. The FGFR Landscape in Cancer: Analysis of 4853 Tumors by Next-Generation Sequencing. Clin. Cancer Res. 2016, 22, 259-267.

18. Tomlinson, D.C.; Knowles, M.A. Altered splicing of FGFR1 is associated with high tumor grade and stage and leads to increased sensitivity to FGF1 in bladder cancer. Am. J. Pathol. 2010, 177, 2379-2386. [CrossRef]

19. Tomlinson, D.C.; Baxter, E.W.; Loadman, P.M.; Hull, M.A.; Knowles, M.A. FGFR1-induced epithelial to

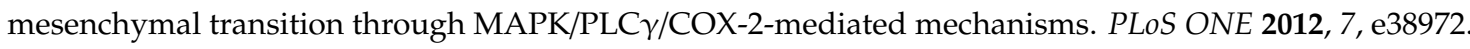
[CrossRef]

20. Cheng, T.; Roth, B.; Choi, W.; Black, P.C.; Dinney, C.; McConkey, D.J. Fibroblast growth factor receptors-1 and -3 play distinct roles in the regulation of bladder cancer growth and metastasis: Implications for therapeutic targeting. PLoS ONE 2013, 8, e57284. [CrossRef]

21. Pal, S.K.; Rosenberg, J.E.; Hoffman-Censits, J.H.; Berger, R.; Quinn, D.I.; Galsky, M.D.; Wolf, J.; Dittrich, C.; Keam, B.; Delord, J.P.; et al. Efficacy of BGJ398, a Fibroblast Growth Factor Receptor 1-3 Inhibitor, in Patients with Previously Treated Advanced Urothelial Carcinoma with FGFR3 Alterations. Cancer Discov. 2018, 8 , 812-821. [PubMed]

22. Pal, S.K.; Bajorin, D.; Dizman, N.; Hoffman-Censits, J.; Quinn, D.I.; Petrylak, D.P.; Galsky, M.D.; Vaishampayan, U.; De Giorgi, U.; Gupta, S.; et al. Infigratinib in upper tract urothelial carcinoma versus urothelial carcinoma of the bladder and its association with comprehensive genomic profiling and/or cell-free DNA results. Cancer 2020, 126, 2597-2606. [PubMed]

23. Katoh, M. FGFR inhibitors: Effects on cancer cells, tumor microenvironment and whole-body homeostasis (Review). Int. J. Mol. Med. 2016, 38, 3-15. [PubMed]

24. Schuler, M.; Cho, B.C.; Sayehli, C.M.; Navarro, A.; Soo, R.A.; Richly, H.; Cassier, P.A.; Tai, D.; Penel, N.; Nogova, L.; et al. Rogaratinib in patients with advanced cancers selected by FGFR mRNA expression: A phase 1 dose-escalation and dose-expansion study. Lancet Oncol. 2019, 20, 1454-1466. [PubMed]

25. Loriot, Y.; Necchi, A.; Park, S.H.; Garcia-Donas, J.; Huddart, R.; Burgess, E.; Fleming, M.; Rezazadeh, A.; Mellado, B.; Varlamov, S.; et al. Erdafitinib in Locally Advanced or Metastatic Urothelial Carcinoma. N. Engl. J. Med. 2019, 381, 338-348. [PubMed]

26. Casalini, P.; Iorio, M.V.; Galmozzi, E.; Menard, S. Role of HER receptors family in development and differentiation. J. Cell Physiol. 2004, 200, 343-350.

27. Eriksson, P.; Sjodahl, G.; Chebil, G.; Liedberg, F.; Hoglund, M. HER2 and EGFR amplification and expression in urothelial carcinoma occurs in distinct biological and molecular contexts. Oncotarget 2017, 8, 48905-48914.

28. Rebouissou, S.; Bernard-Pierrot, I.; de Reynies, A.; Lepage, M.L.; Krucker, C.; Chapeaublanc, E.; Herault, A.; Kamoun, A.; Caillault, A.; Letouze, E.; et al. EGFR as a potential therapeutic target for a subset of muscle-invasive bladder cancers presenting a basal-like phenotype. Sci. Transl. Med. 2014, 6, 244 ra291.

29. Hussain, M.; Daignault, S.; Agarwal, N.; Grivas, P.D.; Siefker-Radtke, A.O.; Puzanov, I.; MacVicar, G.R.; Levine, E.G.; Srinivas, S.; Twardowski, P.; et al. A randomized phase 2 trial of gemcitabine/cisplatin with or without cetuximab in patients with advanced urothelial carcinoma. Cancer 2014, 120, 2684-2693. [CrossRef]

30. Fleischmann, A.; Rotzer, D.; Seiler, R.; Studer, U.E.; Thalmann, G.N. Her2 amplification is significantly more frequent in lymph node metastases from urothelial bladder cancer than in the primary tumours. Eur. Urol. 2011, 60, 350-357. 
31. Kolla, S.B.; Seth, A.; Singh, M.K.; Gupta, N.P.; Hemal, A.K.; Dogra, P.N.; Kumar, R. Prognostic significance of Her2/neu overexpression in patients with muscle invasive urinary bladder cancer treated with radical cystectomy. Int. Urol. Nephrol. 2008, 40, 321-327. [CrossRef] [PubMed]

32. Powles, T.; Huddart, R.A.; Elliott, T.; Jones, R.; Hussain, S.A.; Crabb, S.J.; Ackerman, C.; Jagdev, S.; Chester, J.D.; Hilman, S.; et al. A phase II/III, double-blind, randomized trial comparing maInt.enance lapatinib versus placebo after first line chemotherapy in HER1/2 positive metastatic bladder cancer patients. J. Clin. Oncol. 2015, 33, 4505. [CrossRef]

33. Oudard, S.; Culine, S.; Vano, Y.; Goldwasser, F.; Théodore, C.; Nguyen, T.; Voog, E.; Banu, E.; Vieillefond, A.; Priou, F.; et al. Multicentre randomised phase II trial of gemcitabine+platinum, with or without trastuzumab, in advanced or metastatic urothelial carcinoma overexpressing Her2. Eur. J. Cancer 2015, 51, 45-54. [CrossRef] [PubMed]

34. Ross, J.S.; Wang, K.; Khaira, D.; Ali, S.M.; Fisher, H.A.; Mian, B.; Nazeer, T.; Elvin, J.A.; Palma, N.; Yelensky, R.; et al. Comprehensive genomic profiling of 295 cases of clinically advanced urothelial carcinoma of the urinary bladder reveals a high frequency of clinically relevant genomic alterations. Cancer 2016, 122, 702-711. [CrossRef]

35. Ross, J.S.; Wang, K.; Gay, L.M.; Al-Rohil, R.N.; Nazeer, T.; Sheehan, C.E.; Jennings, T.A.; Otto, G.A.; Donahue, A.; He, J.; et al. A high frequency of activating extracellular domain ERBB2 (HER2) mutation in micropapillary urothelial carcinoma. Clin. Cancer Res. 2014, 20, 68-75.

36. Kamat, A.M.; Dinney, C.P.; Gee, J.R.; Grossman, H.B.; Siefker-Radtke, A.O.; Tamboli, P.; Detry, M.A.; Robinson, T.L.; Pisters, L.L. Micropapillary bladder cancer: A review of the University of Texas M. D. Anderson Cancer Center experience with 100 consecutive patients. Cancer 2007, 110, 62-67. [CrossRef]

37. Kiss, B.; Wyatt, A.W.; Douglas, J.; Skuginna, V.; Mo, F.; Anderson, S.; Rotzer, D.; Fleischmann, A.; Genitsch, V.; Hayashi, T.; et al. Her2 alterations in muscle-invasive bladder cancer: Patient selection beyond protein expression for targeted therapy. Sci. Rep. 2017, 7, 42713. [CrossRef]

38. Braicu, C.; Buse, M.; Busuioc, C.; Drula, R.; Gulei, D.; Raduly, L.; Rusu, A.; Irimie, A.; Atanasov, A.G.; Slaby, O.; et al. A Comprehensive Review on MAPK: A Promising Therapeutic Target in Cancer. Cancers 2019, 11, 1618. [CrossRef]

39. Mendoza, M.C.; Er, E.E.; Blenis, J. The Ras-ERK and PI3K-mTOR pathways: Cross-talk and compensation. Trends Biochem. Sci. 2011, 36, 320-328. [CrossRef]

40. Felsenstein, K.M.; Theodorescu, D. Precision medicine for urothelial bladder cancer: Update on tumour genomics and immunotherapy. Nat. Rev. Urol. 2018, 15, 92-111. [CrossRef]

41. Herrera-Abreu, M.T.; Pearson, A.; Campbell, J.; Shnyder, S.D.; Knowles, M.A.; Ashworth, A.; Turner, N.C. Parallel RNA Int.erference screens identify EGFR activation as an escape mechanism in FGFR3-mutant cancer. Cancer Discov. 2013, 3, 1058-1071. [CrossRef] [PubMed]

42. Wang, L.; Šuštić, T.; Leite de Oliveira, R.; Lieftink, C.; Halonen, P.; van de Ven, M.; Beijersbergen, R.L.; van den Heuvel, M.M.; Bernards, R.; van der Heijden, M.S. A Functional Genetic Screen Identifies the Phosphoinositide 3-kinase Pathway as a Determinant of Resistance to Fibroblast Growth Factor Receptor Inhibitors in FGFR Mutant Urothelial Cell Carcinoma. Eur. Urol. 2017, 71, 858-862. [CrossRef] [PubMed]

43. Datta, J.; Damodaran, S.; Parks, H.; Ocrainiciuc, C.; Miya, J.; Yu, L.; Gardner, E.P.; Samorodnitsky, E.; Wing, M.R.; Bhatt, D.; et al. Akt Activation Mediates Acquired Resistance to Fibroblast Growth Factor Receptor Inhibitor BGJ398. Mol. Cancer Ther. 2017, 16, 614-624. [CrossRef] [PubMed]

44. Bellmunt, J.; Lalani, A.K.A.; Jacobus, S.; Wankowicz, S.A.; Polacek, L.; Takeda, D.Y.; Harshman, L.C.; Wagle, N.; Moreno, I.; Lundgren, K.; et al. Everolimus and pazopanib (E/P) benefit genomically selected patients with metastatic urothelial carcinoma. Br. J. Cancer 2018, 119, 707-712. [CrossRef]

45. Necchi, A.; Lo Vullo, S.; Raggi, D.; Perrone, F.; Giannatempo, P.; Calareso, G.; Togliardi, E.; Nicolai, N.; Piva, L.; Biasoni, D.; et al. Neoadjuvant sorafenib, gemcitabine, and cisplatin administration preceding cystectomy in patients with muscle-invasive urothelial bladder carcinoma: An open-label, single-arm, single-center, phase 2 study. In Urologic Oncology: Seminars and Original Investigations; Elsevier: Amsterdam, The Netherlands, 2018; Volume 36, pp. 8.e1-8.e8. [CrossRef]

46. Shah, C.H.; Pappot, H.; Agerbæk, M.; Holmsten, K.; Jäderling, F.; Yachnin, J.; Grybäck, P.; von der Maase, H.; Ullén, A. Safety and Activity of Sorafenib in Addition to Vinflunine in Post-Platinum Metastatic Urothelial Carcinoma (Vinsor): Phase I Trial. Oncologist 2019, 24, 745. [CrossRef] [PubMed] 
47. Apte, R.S.; Chen, D.S.; Ferrara, N. VEGF in Signaling and Disease: Beyond Discov.ery and Development. Cell 2019, 176, 1248-1264. [CrossRef]

48. Ferrara, N.; Adamis, A.P. Ten years of anti-vascular endothelial growth factor therapy. Nat. Rev. Drug Discov. 2016, 15, 385-403.

49. Hahn, N.M.; Stadler, W.M.; Zon, R.T.; Waterhouse, D.; Picus, J.; Nattam, S.; Johnson, C.S.; Perkins, S.M.; Waddell, M.J.; Sweeney, C.J.; et al. Phase II trial of cisplatin, gemcitabine, and bevacizumab as first-line therapy for metastatic urothelial carcinoma: Hoosier Oncol.ogy Group GU 04-75. J. Clin. Oncol. 2011, 29, 1525-1530. [CrossRef]

50. Rosenberg, J.E.; Ballman, K.V.; Halabi, S.; Watt, C.; Hahn, O.M.; Steen, P.D.; Dreicer, R.; Flaig, T.W.; Stadler, W.M.; Sweeney, C.; et al. CALGB 90601 (Alliance): Randomized, double-blind, placebo-controlled phase III trial comparing gemcitabine and cisplatin with bevacizumab or placebo in patients with metastatic urothelial carcinoma. J. Clin. Oncol. 2019, 37, 4503. [CrossRef]

51. Yakes, F.M.; Chen, J.; Tan, J.; Yamaguchi, K.; Shi, Y.; Yu, P.; Qian, F.; Chu, F.; Bentzien, F.; Cancilla, B.; et al. Cabozantinib (XL184), a novel MET and VEGFR2 inhibitor, simultaneously suppresses metastasis, angiogenesis, and tumor growth. Mol. Cancer Ther. 2011, 10, 2298-2308. [CrossRef]

52. Nadal, R.; Mortazavi, A.; Stein, M.; Pal, S.K.; Davarpanah, N.; Parnes, H.L.; Ning, Y.M.; Cordes, L.M.; Lin, J.; Bagheri, M.; et al. Final results of a phase I study of cabozantinib (cabo) plus nivolumab (nivo) and cabonivo plus ipilimumab (Ipi) in patients (pts) with metastatic urothelial carcinoma (mUC) and oTher. genitourinary (GU) malignancies. Ann. Oncol. 2017, 28, v295. [CrossRef]

53. Nadal, R.M.; Mortazavi, A.; Stein, M.; Pal, S.K.; Davarpanah, N.N.; Parnes, H.L.; Ning, Y.M.; Cordes, L.M.; Bagheri, M.H.; Lindenberg, L.; et al. Results of phase i plus expansion cohorts of cabozantinib (Cabo) plus nivolumab (Nivo) and CaboNivo plus ipilimumab (Ipi) in patients (PTS) with with metastatic UROTHELIAL CARCINOMA (mUC) and oTher. genitourinary (GU) malignancies. J. Clin. Oncol. 2018, 36, 515. [CrossRef]

54. Necchi, A.; Giannatempo, P.; Raggi, D.; Anichini, A.; Calareso, G.; Crippa, F.; Mariani, L. Cabozantinib $(\mathrm{CABO})$ plus durvalumab (DURVA) in patients with advanced and chemoTher.apy-treated bladder carcinoma of urothelial and non-urothelial histology: An open-label, single-arm, phase 2 trial. J. Clin. Oncol. 2018, 36, TPS536. [CrossRef]

55. Raggi, D.; Giannatempo, P.; Anichini, A.; Calareso, G.; Crippa, F.; Mariani, L.; Necchi, A. Cabozantinib (CABO) plus durvalumab (DURVA) in patients with advanced and chemoTher.apy-treated bladder carcinoma, of urothelial and non-urothelial histology: The open-label, single-arm, phase 2 ARCADIA trial. Eur. Urol. Suppl. 2018, 17, e1150. [CrossRef]

56. Maia, M.C.; Agarwal, N.; McGregor, B.A.; Vaishampayan, U.N.; Choueiri, T.K.; Green, M.C.; Hessel, C.; Scheffold, C.; Schwab, G.; Powles, T.; et al. Phase 1b trial of cabozantinib in combination with atezolizumab in patients with locally advanced or metastatic urothelial carcinoma (UC) or renal cell carcinoma (RCC). J. Clin. Oncol. 2018, 36, TPS42. [CrossRef]

57. Pal, S.K.; Vaishampayan, U.N.; Castellano, D.E.; Necchi, A.; Van Herpen, C.M.L.; Ramsingh, G.; Loriot, Y.; Agarwal, N. Phase lb (COSMIC-021) trial of cabozantinib (C) in urothelial carcinoma (UC) or C in combination with atezolizumab (A) in patients (pts) with UC, castrate resistant prostate cancer (CRPC) or renal cell carcinoma (RCC). J. Clin. Oncol. 2019, 37, TPS683. [CrossRef]

58. Powles, T.; Choueiri, T.K.; Agarwal, N.; Necchi, A.; Loriot, Y.; McKay, R.R.; Chang, P.Y.; Kondo, A.; Wang, E.; McGregor, B.; et al. Phase $1 \mathrm{~b}$ Trial of Cabozantinib in Combination with Atezolizumab in Patients with Locally Advanced or Metastatic Renal Cell Carcinoma and Urothelial Carcinoma. Eur. Urol. Suppl. 2017, 16, e2789. [CrossRef]

59. Agarwal, N.; Loriot, Y.; McGregor, B.A.; Dreicer, R.; Dorff, T.B.; Maughan, B.L.; Kelly, W.K.; Pagliaro, L.C.; Srinivas, S.; Squillante, C.M.; et al. Cabozantinib (C) in combination with atezolizumab (A) in patients (pts) with metastatic castration-resistant prostate cancer (mCRPC): Results of Cohort 6 of the COSMIC-021 Study. J. Clin. Oncol. 2020, 38, 139. [CrossRef]

60. O’Mahony, D.; Bishop, M.R. Monoclonal antibody Therapy. Front. BioSci. 2006, 11, 1620-1635. [CrossRef]

61. Lucas, A.T.; Price, L.S.L.; Schorzman, A.N.; Storrie, M.; PiScitelli, J.A.; Razo, J.; Zamboni, W.C. Factors Affecting the Pharmacology of Antibody-Drug Conjugates. Antibodies 2018, 7, 10. [CrossRef]

62. Khongorzul, P.; Ling, C.J.; Khan, F.U.; Ihsan, A.U.; Zhang, J. Antibody-Drug Conjugates: A Comprehensive Review. Mol. Cancer Res. 2020, 18, 3-19. [CrossRef] 
63. Tsuchikama, K.; An, Z. Antibody-drug conjugates: Recent advances in conjugation and linker chemistries. Protein Cell 2018, 9, 33-46. [CrossRef] [PubMed]

64. Petrylak, D.P.; Balar, A.V.; O’Donnell, P.H.; McGregor, B.A.; Heath, E.I.; Yu, E.Y.; Galsky, M.D.; Hahn, N.M.; Gartner, E.M.; Pinelli, J.; et al. EV-201: Results of enfortumab vedotin monotherapy for locally advanced or metastatic urothelial cancer previously treated with platinum and immune checkpoint inhibitors. J. Clin. Oncol. 2019, 37, 4505. [CrossRef]

65. Hanna, K.S. Clinical Overview of Enfortumab Vedotin in the Management of Locally Advanced or Metastatic Urothelial Carcinoma. Drugs 2020, 80, 1-7. [CrossRef] [PubMed]

66. Gong, J.; Shen, L.; Wang, W.; Fang, J. Safety, pharmacokinetics and efficacy of RC48-ADC in a phase I study in patients with HER2-overexpression advanced solid cancer. J. Clin. Oncol. 2018, 36, e16059. [CrossRef]

67. Sheng, X.; Zhou, A.-P.; Yao, X.; Shi, Y.; Luo, H.; Shi, B.; Liu, J.; Yu, G.; He, Z.; Hu, C.; et al. A phase II study of RC48-ADC in HER2-positive patients with locally advanced or metastatic urothelial carcinoma. J. Clin. Oncol. 2019, 37, 4509. [CrossRef]

68. Li, B.T.; Makker, V.; Buonocore, D.J.; Offin, M.D.; Olah, Z.T.; Panora, E.; Shen, R.; Ho, A.L.; Yaeger, R.; Iyer, G.; et al. A multi-histology basket trial of ado-trastuzumab emtansine in patients with HER2 amplified cancers. J. Clin. Oncol. 2018, 36, 2502. [CrossRef]

69. Strzebonska, K.; Waligora, M. Umbrella and basket trials in Oncol.ogy: Ethical challenges. BMC Med. Ethics 2019, 20, 58. [CrossRef]

70. Bahleda, R.; Italiano, A.; Hierro, C.; Mita, A.; Cervantes, A.; Chan, N.; Awad, M.; Calvo, E.; Moreno, V.; Govindan, R.; et al. Multicenter Phase I Study of Erdafitinib (JNJ-42756493), Oral Pan-Fibroblast Growth Factor Receptor Inhibitor, in Patients with Advanced or Refractory Solid Tumors. Clin. Cancer Res. 2019, 25, 4888-4897. [CrossRef]

71. Faltas, B.M.; Prandi, D.; Tagawa, S.T.; Molina, A.M.; Nanus, D.M.; Sternberg, C.; Rosenberg, J.; Mosquera, J.M.; Robinson, B.; Elemento, O.; et al. Clonal evolution of chemoTher.apy-resistant urothelial carcinoma. Nat. Genet. 2016, 48, 1490-1499. [CrossRef]

72. Nordentoft, I.; Lamy, P.; Birkenkamp-Demtröder, K.; Shumansky, K.; Vang, S.; Hornshøj, H.; Juul, M.; Villesen, P.; Hedegaard, J.; Roth, A.; et al. Mutational context and diverse clonal development in early and late bladder cancer. Cell Rep. 2014, 7, 1649-1663. [CrossRef] [PubMed]

73. Lamy, P.; Nordentoft, I.; Birkenkamp-Demtröder, K.; Thomsen, M.B.; Villesen, P.; Vang, S.; Hedegaard, J.; Borre, M.; Jensen, J.B.; Høyer, S.; et al. Paired Exome Analysis Reveals Clonal Evolution and Potential Ther.apeutic Targets in Urothelial Carcinoma. Cancer Res. 2016, 76, 5894-5906. [CrossRef] [PubMed]

74. Thomsen, M.B.; Nordentoft, I.; Lamy, P.; Høyer, S.; Vang, S.; Hedegaard, J.; Borre, M.; Jensen, J.B.; Ørntoft, T.F.; Dyrskjøt, L. Spatial and temporal clonal evolution during development of metastatic urothelial carcinoma. Mol. Oncol. 2016, 10, 1450-1460. [CrossRef] [PubMed]

75. Birkenkamp-Demtröder, K.; Christensen, E.; Nordentoft, I.; Knudsen, M.; Taber, A.; Høyer, S.; Lamy, P.; Agerbæk, M.; Jensen, J.B.; Dyrskjøt, L. Monitoring Treatment Response and Metastatic Relapse in Advanced Bladder Cancer by Liquid Biopsy Analysis. Eur. Urol. 2018, 73, 535-540. [CrossRef] [PubMed]

76. Christensen, E.; Birkenkamp-Demtröder, K.; Sethi, H.; Shchegrova, S.; Salari, R.; Nordentoft, I.; Wu, H.-T.; Knudsen, M.; Lamy, P.; Lindskrog, S.V.; et al. Early Detection of Metastatic Relapse and Monitoring of Ther.apeutic Efficacy by Ultra-Deep Sequencing of Plasma Cell-Free DNA in Patients With Urothelial Bladder Carcinoma. J. Clin. Oncol. 2019, 37, 1547-1557. [CrossRef] [PubMed]

77. Christensen, E.; Birkenkamp-Demtröder, K.; Nordentoft, I.; Høyer, S.; van der Keur, K.; van Kessel, K.; Zwarthoff, E.; Agerbæk, M.; Ørntoft, T.F.; Jensen, J.B.; et al. Liquid Biopsy Analysis of FGFR3 and PIK3CA Hotspot Mutations for Disease Surveillance in Bladder Cancer. Eur. Urol. 2017, 71, 961-969. [CrossRef] [PubMed]

78. Vandekerkhove, G.; Todenhöfer, T.; Annala, M.; Struss, W.J.; Wong, A.; Beja, K.; Ritch, E.; Brahmbhatt, S.; Volik, S.V.; Hennenlotter, J.; et al. Circulating Tumor DNA Reveals Clinically Actionable Somatic Genome of Metastatic Bladder Cancer. Clin. Cancer Res. 2017, 23, 6487-6497. [CrossRef] [PubMed] 